\title{
XXXII. A new improved type of chronograph
}

\section{Robert Ludwig Mond M.A. F.R.S.E. \& Meyer Wilderman Ph.D. B.Sc.}

To cite this article: Robert Ludwig Mond M.A. F.R.S.E. \& Meyer Wilderman Ph.D. B.Sc. (1906) XXXII. A new improved type of chronograph , Philosophical Magazine Series 6, 11:63, 393-402, DOI: $10.1080 / 14786440609463455$

To link to this article: http://dx.doi.org/10.1080/14786440609463455

\section{Published online: 16 Apr 2009.}

Submit your article to this journal

山 Article views: 3 
XXXII. A New Improved Type of Chronograph. By Robert Ludwig Mond, M.A., F.R.S.E., and Meyer Wilderman, Ph.D., B.Sc.*

[Plate VIII.]

IN the course of a physico-chemical investigation we made some years ago a chronograph measuring time to $0 \cdot 1$ second was required. We procured one of the best make with a revolving drum of $60 \mathrm{~cm}$. circumference making one revolution per minute, and subjected it to a careful investigation. We found on calibrating it with a clock making electrical contacts automatically every minute, that not only was the absolute value of each revolution not equal to a minute, but that the differences in time between two contacts proved to be very irregular and greatly different. On further comparing four curves obtained successively one after another with the same instrument, the clock having been wound up afresh overy time, we found that the form and shape of the succession of the contacts of each spiral was not always the same, so that no correction table could be applied in connexion with the above instrument. Since it is exceedingly difficult to get a large revolving drum to move at the above speed with great regularity, it was evident that unless a simultaneous calibration of the curve is made for every second or two seconds, the above type of instrument cannot be used even for moderately accurate measurements of time.

Since no fault could be found with the workmanship of the instrument, it was evident that the reasons for these irregularities bad to be looked for in the principle upon which the instrument for high speeds was constructed. This it was not difficult to discover. It is a well-known fact that very little is required to stop a clock, and still less to make its movements irregular. By making a clock drive a drum with a speed of one revolution per minute instead of 24 hours, the clock has to do about 1000 to 1500 times more work, and the solution of the problem for chronographs of higher speed could therefore be only effected if this enormous increase of work of the clock could be correspondingly counterbalanced by a reduction in the work the clock has to do per each revolution.

The principles upon which our chronograph was worked out can be summarized as follows:-(1) Instead of moving the heavy drum, thick screw, and the one or two carriages

* Communicated by the Authors.

Phil. Mag. S. 6. Vol. 11. No. 63. March 1906. 
with writing-pens, we keep them all stationary and only move the light spindle with the writing-pen, thus diminishing the load upon the clock arising from the work transmitted by the clock. (2) We reduce the friction of the different parts in motion to that of the moving spindle only, and further reduce that by the use of friction wheels or balls, \&c. (3) We avoid the reactions of inertia, as far as possible, by a perfect balance of the moving part of the instrument-the spindle.

Experiments for several years showed the intricacy and the minuteness of conditions to be carried out simultaneously in the construction of a chronograph for high speeds before a satisfactory result could be obtained. We shall for this reason describe and explain all those details upon which the successful construction and use of our chronograph depends.

Figure I. (\& Pl. VIII. fig. 1) represents a horizontal type of

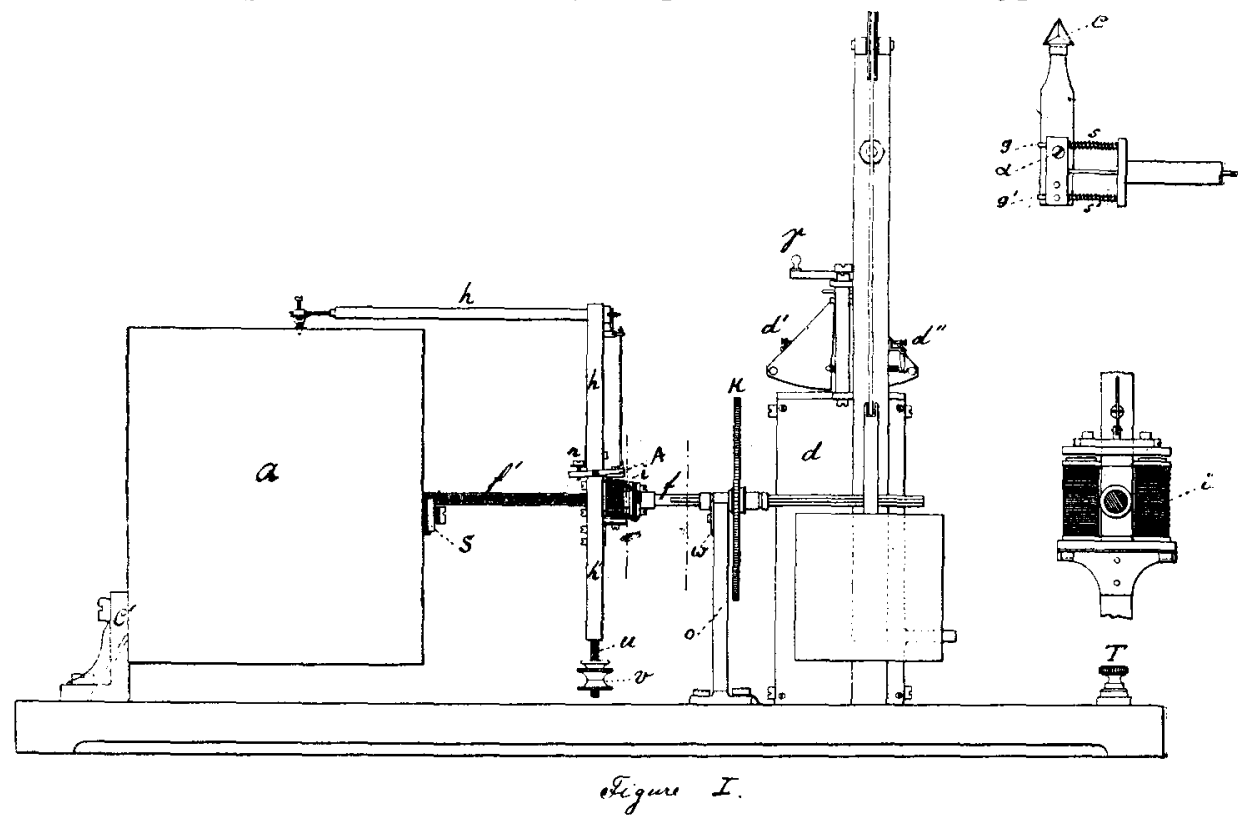

our chronograph, as made for us by Messrs. Sanger, Shepherd and Co. $d$ is the driving clock, $a$ is the stationary drum $60 \mathrm{~cm}$. circumference fixed (in exact horizontal position) to the bracket $\left(\mathrm{C}^{\prime}\right)$. The horizontal spindle $(f)$ carrying the pen$\operatorname{arm}(h)$ of the writing-pen $(c)$ and the electromagnet $(i)$ is counterbalanced by the nut $(v)$ which is adjustable on the screw $(u)$. The horizontal spindle passes at one ead through the boss of a pinion wheel (K), driven by the wheel $(l)$ 
of the clock, thus describing a circle round the drum. A strip projecting from the spindle forms a long feather, and a corresponding featherway in the boss of the pinion, $2 \mathrm{~mm}$. wide, renders the spindle capable of axial movement with regard to the pinion, caused by the screw of the spindle $\left(f^{\prime}\right)$ and the half-nut (S), fixed to the drum and insulated from the same by means of the ebonite strip. Owing to this double action of the spindle, the writing-pen describes a spiral on the drum. The instrument shown in fig. I. gives a spiral $50 \times 60=3000 \mathrm{~cm}$. long, the circumference of the drum being $60 \mathrm{~cm}$. Since the writing pen can easily make one revolution per minute or in 10 seconds, we have $10 \mathrm{~mm}$. or $60 \mathrm{~mm}$. for registration of one second. The line of our pen being very thin, we can read well to $0.2 \mathrm{~mm}$. or even less, i. e. 1/50 or 1/300 of a second, provided, however, means are found to make electrical contacts to the same degree of accuracy; a problem which, it seems to us, will give much trouble if a conscientious scientific investigation should be properly attempted. Since in our type of chronograph the drum is stationary and a great extension in its height and width means only an extension of the length of the arm of the spindle, which means only a small increase of load and almost no increase in the friction or the reaction of inertia, we are thus able to construct instruments with still greater length of spiral and of the same accuracy.

The boss of the spindle rests on two little friction wheels $(w)$ fixed to the support (o), and is kept in position by the slight exterior circumferential groove on the boss with which the friction wheels engage; the friction of the spindle at this end is thus reduced to two points only. The screw end of the spindle has only to guide the pen which writes with almost no friction (the spindle must be naturally placed exactly in the centre of the drum). It is made thin for this reason, and the half-nut (s) contains only a little more than a line of the thread just sufficient to move the spindle securely forward. In this manner the friction at this end of the spindle was also reduced to the smallest possible amount.

It will be perceived that the wheel $(\mathrm{K})$ is not fixed to the spindle. This enables us to exchange without trouble one pair of wheels for another giving a sinaller or greater speed (say one revolution of the spindle in one minute or in ten seconds), so as to get the speed which is most suitable for the investigation of the given physical, physico-chemical, physiological or meteorological phenomenon, \&c. To allow of the use of the exchange wheels an adjustable fly is provided for the instrument. 
Our adjustable fly is an improved type of Breguet governor. Its purpose is a double one : first, to counteract all small irregularities of speed; secondly, to adjust the absolute value of the time of each revolution when different pairs of wheels are used or when the springs in the natural course of time vary in elasticity. As to the first purpose, it should be remarked that only when the irregularities in the movement of the clock independent of the governor are very small, is the governor effective; when the variations are too great the governor serves no purpose and is a decoration only. The adjustment of the absolute values of the speed of each revolution is usually made as follows. When the wheels, \&c. of the instrument are properly chosen, the final adjustment is usually effected by employing flexible spirals of a given material and of a certain number of turnings. This adjustment can, however, only be a rough one, and what is still worse, even if a perfect adjustment is once effected it is certain not to remain perfect after being in use for some time owing to the variation in the flexibility of the springs, to the effect of slow oxidation, moisture, fatigue, \&c. So also when exchangewheels are used, the speed is not in proportion to the number of teeth in the wheel, as is often assumed, becanse the work to be spent in moving the clock parts and the spindle is not directly proportional to the speed of each revolution; i.e., each pair of wheels must bave its own governor which is to be specially arranged for the given speed. To meet all the above requirements, the springs of the governor are fixed as shown in fig. II. (p. 399), and by turning the screws $d^{\prime}$ and $d^{\prime \prime}$ they can be considerably increased or diminished in tension; the adjustment of speed can therefore be carried out with great sensitiveness and without difficulty.

At the end of the cross arm $(h)$ a screw $(u)$ is arranged, which together with the nut $(v)$ forms a balance-weight at the end of the cross arm. This arrangement gives an adjustable and extremely accurate means for securing an exact balance of all parts of the spindle while in position, which, besides the clock, is the only movable part of the instrument. This is also a point of great consideration in chronographs of great speed, because greater speed does not necessarily mean a corresponding greater accuracy. When a clock moves different parts of an instrument, not only does the clock move those parts, but those parts also move the clock. Since it is exceedingly difficult to get the wall of a hollow cylinder of a uniform thickness throughout, it being a sufficiently difficult task to get its upper surface true alone, the drum is never or almost never completely counterbalanced 
in all its parts; its movement is, owing to inertia, for this reason not of a uniform character, and this upsets the regularity of the movement of the clock. In our instrument the parts $f$ and $f^{\prime}$ themselves form the axis, and the two cross-pieces ( $h$ and $h^{\prime}$ ) with the nut $(v)$ form the arms of a balance carrying the weights to be adjusted to each other by means of the screw and nut. It is of no little importance for the accuracy of the spiral obtained, that in our instrument this result is produced by one moving part only, while in other instruments it is produced by several moving parts, which in the nature of things can never be made to be absolutely dependent one upon another.

We now pass to the description of our improved means for operating and working the writing-pen. After many trials and modifications the following arrangement was adopted. A bell-crank is provided at the end of the cross arm, one of its arms being connected by a rigid link to the armature (A) of the magnet, while the other is connected in a similar manner through the axial hole in the pen-arm to the penholder itself. The end of the pen-arm carries a small cross-head from which two guides ( $g$ and $g^{\prime}$ ) parallel to the arm project. A cross-piece to the centre of which the operating link is connected slides on these guides, its motion being controlled by light flexible springs $\left(s\right.$ and $s^{\prime}$ ), and to the cross-piece a flexible strip carrying the pen is secured. The pressure with which the pen bears upon the drum is regulated by a small screw ( $\alpha$ ) acting between the flexible strip and the cross-piece. In our newest type of chronograph arrangements are made to raise the pen from the drum, when the paper is changed, so that the pressure of the pen upon the drum, once adjusted, should always remain absolutely the same. For this purpose a little plate carrying the writing-pen with the screw is hinged to the cross-piece. This arrangement provides a very steady support for the pen, making vibrations of the penholder impossible and keeping the pen in all positions of the spindle at exactly the same distance from the drum (instead of resting on it), and this enables the pen to be brought just so near to the drum as is necessary to trace a spiral on the same, i.e. with the least possible friction. To the arm carrying the pen a reservoir containing a considerable quantity of ink may be fixed, from which the ink flows into the pen through a capillary tube, or a capillary tube containing a cotton thread. By this means the pen never becomes dry, and there is no necessity for repeated cleaning and filling with ink.

The writing pen is actuated electrically. The current 
enters the terminal ( $\mathrm{T}$ ), which is in electrical coniact with the base of the instrument, it passes through the support to the friction wheels, driving spindle, electromagnet, the screw, and then, by way of the insulated half-nut, from here it passes by the return wire to the terminal $\left(\mathrm{T}^{\prime}\right)$ which is insulated from the base of the instrument.

There is another distinctive feature in the arrangement of our pen for registration, and this is that our pen can be used at the same time both for registration and calibration. The length of the two guides $\left(g\right.$ and $\left.g^{\prime}\right)$, the greater flexibility of the two flexible springs ( $s$ and $s^{\prime}$ ), allow the pen to slide on the guides long distances, and for this the electromagnet (i) must also be adjusted at its screw $(n)$. By connecting the terminals ( $T$ and $T^{\prime}$ ) with a battery, and by using the pendulum of a clock or other suitable calibrating arrangements in the circuit for making contacts, we are able by inserting a corresponding resistance to adjust the current passing through the electromagnet so as to get for calibration small deviations of the pen from the normal spiral as desired each second, or better, each half minute or minute. By having the terminals ( $\mathrm{T}$ and $\mathrm{T}^{\gamma}$ ) at the same time connected with another battery sending the current in the same direction, and by having the electrical key or the automatic contact of the rain-gauge, anemometer, speed indicator, \&c., we can so adjust the voltage and the resistance as to get a greater current through the electromagnet and a correspondingly greater deviation of the writing-pen from its normal course, thus registering the observed or recorded phenomenon at the same time. The results obtained for calibration and registration cannot possibly be mistaken for one another even if both contacts should happen to be effected simultaneously, because two intervals of calibration cannot possibly with our instrument be mistaken for one, and the longer line of registration cannot be covered by the shorter one of calibration. Not only does this dispense with a second carriage and pen, but also leads to more accurate results, because we register and calibrate straight on one and the same spiral, not on two separate ones, and we have not subsequently to transfer the calibration from one spiral to the other. For the special purposes, where two different kinds of phenomenon are to be recorded simultaneously, e.g. wind and rain, or where two observers have to record simultaneously the same phenomenon, such as the transit of a star in astronomical work, the instrument can be provided with two pens.

The great regularity and perfection of motion of the spindle 
in our chronograph attained in the manner described above is best seen from the fact that not the least sound (except from the fly) can be heard when the instrument is working, while in instruments of the older type of a similar speed harsh sounds both in the clock and in other parts of the instrument are heard, indicative of the irregularity of motion.

In figure II. (\& Pl. VIII. fig. 2) the vertical type of our chronograph is given. The principles and details are the

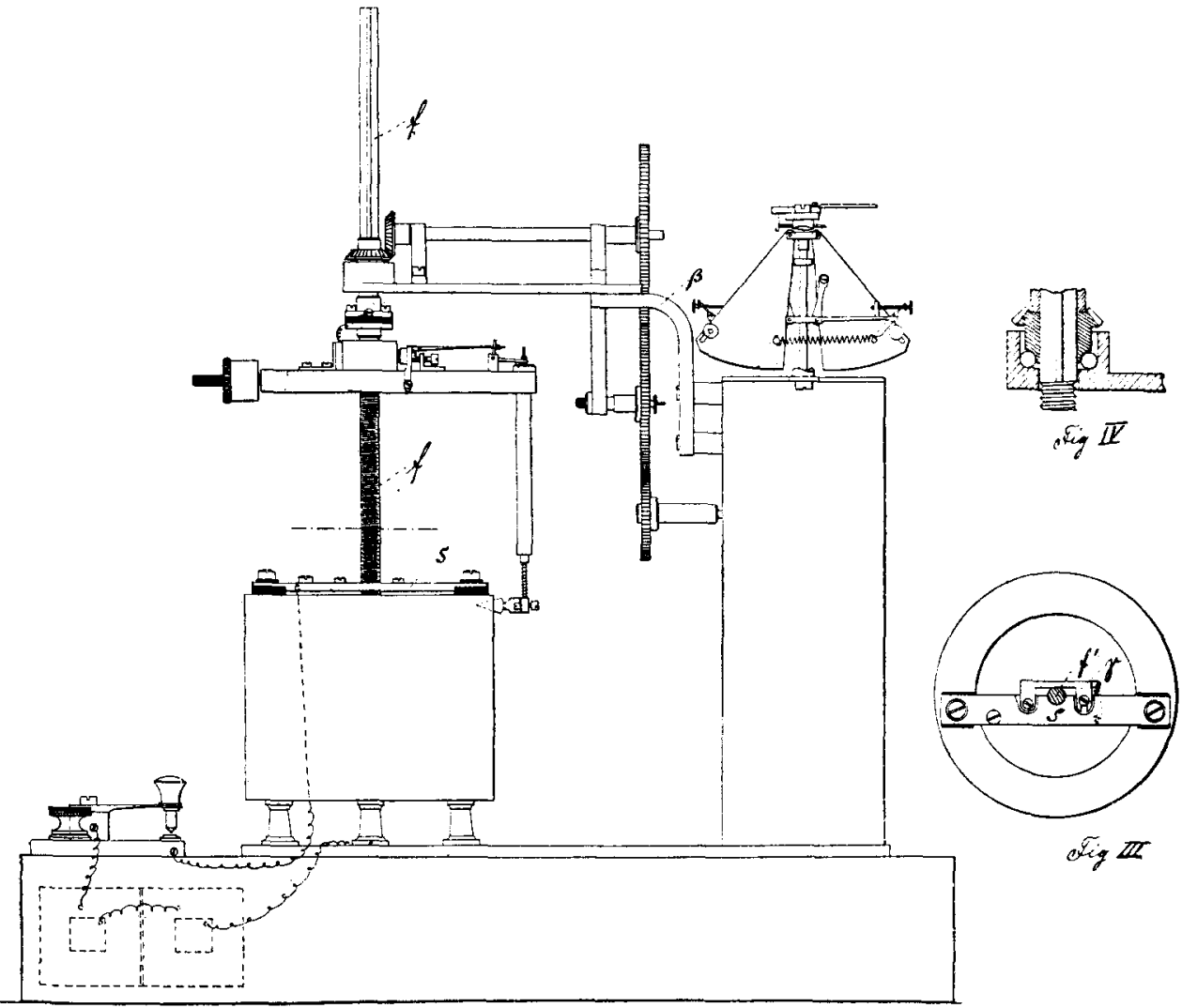

$\operatorname{Prg} I I$

same here as in the horizontal type and are indicated by the same letters. Here the spindle is arranged with its driving end upwards, the weight of the pen-carrying system being taken by the half nut (S) on the drum.

A detail of this portion is given in figure III., a hinged 
clip $(\gamma)$, provided with spring and clamping screw, serving to hold the spindle $\left(f^{\prime}\right)$ in operative engagement with the half-nut. The driving is effected through spur-wheels, supported by the bracket $(\beta)$; the bevel-wheel, as shown in detail in figures II., III., and IV., being arranged to run on ball bearings. The arrangements of the pen-carrying system are similar to that described with reference to the horizontal type of instrument, the electrical connexions being also substantially the same.

The vertical type is an improvement on the horizontal type, though usually made of a smaller size, since it allows continuous reading of the records on the drum, can stand more knocking about, is easier to be accurately constructed, and gives also better results.

When the whole spiral is completed, the instrument stops automatically by itself. In the horizontal type we raise then the spindle $\left(f f^{\prime}\right)$ from the half-nut $(s)$, and push it through the boss of pinion $(\mathrm{K})$ back to its former position, stopping first the governor by means of $\left(\gamma^{\prime}\right)$. In the vertical type, we open clip $(\gamma)$, release the screw of the spindle from the half-nut, and push it up to its former position.

Both types are set into motion or stopped, whenever desired, by stopping or releasing the governor by means of $\gamma^{\prime}$.

While costly and complicated arrangements for calibration each second are indispensable for instruments with revolving drums, all we require for very accurate work with our instrument where the movement of the pen is very regular is to calibrate it, using an ordinary $\frac{1}{5}$ sec. or $\frac{1}{10}$ sec. watch and electric key at longer intervals, say each minute (at the same time as we make our research observations), and in this case we do not even require to exactly adjust the absolute value of a revolution, having only to use a factor. The same or almost the same error in calibration falls upon a minute instead of upon a second, and the results obtained in this manner are much more accurate and satisfactory. Since no work can be done for nothing, any extra work of the pen spent on calibration is not conducive to the accuracy of the resulis obtained, and it is well that we are able to avoid frequent calibration.

Up to the present we have tried a great number of arrangements for calibration, some of them very complicated and difficult. The great accuracy and regularity in the movement of the writing-pen, as well as the great length of the spiral, make our vertical instrument a very handy and easily manageable recording calibrator for other instruments 
with revolving drums, where those have to be used in view of the nature of the research (physiological, physical, \&c.) in spite of their inaccuracy. All that is required for this is to fix to the axis of the revolving drum a suitable wheel with projections making electrical contacts for our chronograph, at as frequent intervals as is desirable, and to calibrate the value of each revolution of the pen of our instrument with an ordinary watch, at a greater time interval.

The accuracy of the instrument as a time indicator and the length of the spiral allows of its wide application for all sorts of scientific and technical purposes: e.g. by a corresponding variation in the accessory instruments for more frequent contacts a considerably more accurate anemometer, speed indicator, \&c., is easily arranged, allowing a very much greater accuracy than has hitherto been practicable. We shall illustrate this only on the two instruments mentioned, i.e. on the anemometer and the speed indicator.

The anemometer has to indicate the miles of wind in the unit of time, i.e. $\frac{\mathrm{F}}{t}$. In the present instrument two clocks are usually used : one moves the drum with the speed of one revolution in 24 hours (this gives $t$ ) ; the other raises the writing-pen and allows it to drop, thus extending the line for contacts several times (this gives F). Since the length of the curve for time is very short, the registration of $t$ is very defective, $\frac{\mathrm{F}}{t}$ must be defective also. With our instrument the revolving pen makes a contact on our spiral for say each $\mathrm{F}$ miles of wind. The spiral is about 50 times longer than with ordinary instruments and is used both for the indication of time and for the contacts. The length of line is about 10 times longer for $\mathrm{F}$ and about 50 times longer for $t$.

The speed indicator has to give the number of revolutions (of an electromagnet, dynamo, gas-engine, steam-engine, motor car, \&c.) in the unit of time, i.e. $\frac{\mathrm{S}}{t}$. In all present instruments time is given by a revolving drum. The indication of time $(t)$ is very wrong, especially where large strips of paper are rolled and unrolled from drums and drawn by a clock (in the first instance on account of enormous friction). The number of revolutions is given by a rising fly-this $(s)$ is again wrong, because the rise of the fly is not directly proportional to the speed. Then the pen writes not in straight lines, but in ares. The result is that we get an idea of the speed, but no quantitative measurement of it, and we have no means of knowing exactly the speed at any given 
time owing to the interference of too many sources of error. With our instrument we arrange that a contact should be made by the dynamo, gas-engine, motor car, \&c. \&c., at convenient intervals, say each 1 or 10 or 100 revolutions. The distances between the contacts of the spiral on paper ruled in $\mathrm{mm}$. to indicate the time gives also at a glance the character of the varying speed, but the same unit divided by the distances between two contacts read in mm., gives at the same time also exactly the speed between the time intervals indicated on the paper. The greater length of spiral also allows of this variation being observed more frequently and with greater accuracy.

I Jondon, August 1905.

XXXIII. The Comparison of Electric Fields by means of an Oscillating Electric Needle. By David OWen, B.A. (Camb.), B.Sc.(Lond.), Lecturer in Plyysics, Birkleck College, London*.

TXPERIMENT's on fields of force due to electric charges 1 suffer as compared with the corresponding measurements in magnetic fields by reason of the impracticability of obtaining an "electric needle" corresponding to the oscillating magnetic needle. But while it is true that a permanently electrified needle cannot be obtained, it is possible to have one that has equal charges of opposite sign induced by the field. This paper contains an aecount of experiments on the use of such a needle for the measurement of electric fields, steady or alternating, and for the experimental illustration of some of the laws of electrostatics.

\section{Theory.}

Consider a cylindrical needle supported at its centre by a fibre whose torsional control is negligible, placed with its axis horizontal in a uniform horizontal field of strength $F$. The needle will move so as to set itzelf parallel to the field. If disturbed from that position a restoring couple will come into play. This couple will be proportional, both to the induced charge, i. e. to the "pole-strength," and to the field. As the former is proportional to the latter, it follows that the couple will vary as the square of the field-strength. It will diminish as the angle between the axis of the needle and the direction of the field increases, and will clearly be zero when the two are at right angles. In the case of an

* Communicated by the Physical Society : read June 30, 1905. 


$$
\frac{E}{L}
$$

